\title{
Gig economy en Iberoamérica: caracterización del mercado laboral online de los profesionales de TI freelancers
}

Revista Latinoamericana de Economía y Sociedad Digital

Issue 2, agosto 2021

Autores: $\underline{E \text { dna Patricia Santiago Vargas }}(\mathbb{D}$, Catalina Ovando $(\mathbb{D}$

DOI: $10.53857 /$ YYDX3524

Publicado: 25 agosto, 2021

Recibido: 15 marzo, 2021

Cita sugerida: Santiago Vargas, Edna Patricia \& Ovando, Catalina (2021) "Gig economy en Iberoamérica: caracterización del mercado laboral online de los profesionales de TI freelancers" en Revista Latinoamericana de Economía y Sociedad Digital, Issue 2

Licencia: Creative Commons Atribución-NoComercial 4.0 Internacional (CC BY-NC 4.0)

Tipo: Estudio de caso

Palabras clave: freelancers, gig economy, mercado laboral, plataformas digitales, Workana

\section{Resumen}

En los últimos años se ha observado un incremento en el uso de plataformas digitales de trabajo freelance (Freelancer.com 2015; 2019) como alternativa al empleo tradicional. Sin embargo, existe un conocimiento limitado sobre las opciones de trabajo que tienen los profesionales de TI dentro de estas plataformas. El objetivo de este documento es presentar una caracterización del mercado laboral de los profesionales de las Tecnologías de la Información (TI) que se emplean en la gig economy mediante la plataforma Workana. Para este fin se recopilaron ofertas de trabajo pertenecientes a la categoría "Programación y tecnología", publicadas de agosto a diciembre de 2019 por empleadores localizados en Argentina, Brasil, Chile, Colombia, México y España. Se contempló el estado de desarrollo, alcance, presupuesto, subcategoría y habilidades por cada oferta. Para conocer las habilidades mejor remuneradas se consideraron aquellas ofertas asociadas a una sola habilidad. Se encontró que las habilidades más demandadas por los empleadores fueron PHP, JavaScript, MySQL, Android, HTML, HTML5, Responsive Web Design, iPhone, CSS, 
API y WordPress. Las habilidades mejor remuneradas fueron Android, C, React native y React.js. Se espera que los resultados planteados sirvan como referente para que los trabajadores autónomos de TI en Iberoamérica conozcan las habilidades mejor retribuidas a través de las plataformas digitales de empleo independiente. Así mismo, se espera que la academia y los gobiernos consideren estos hallazgos para alinear los programas de capacitación a la demanda laboral presente en la gig economy.

\section{Abstract}

In recent years, it has been noted an increase in the use of digital platforms for freelance jobs (Freelancer.com 2015; 2019) as an alternative to the traditional employment. Nevertheless, there is limited knowledge of the options IT professionals have in these platforms. The aim of this document is to present a characterization of the labor market of the Information Technology (IT) professionals that are employed in the gig economy through the platform Workana. For this purpose, we have gathered some job offers belonging to the category "Programing and Technology", published from August to December, 2019, by employers located in Argentina, Brazil, Chile, Colombia, Mexico and Spain. We considered the state of development, scope, budget, subcategory and skills for each offer. To learn about the highest paying skills we have considered the offers associated to only one skill. The skills that are most demanded by the employers were PHP, JavaScript, MySQL, Android, HTML, HTML5, Responsive Web Design, iPhone, CSS, API and WordPress. The best paying skills were Android, C, React native and React.js. The aim of the results foreseen is to be considered as a point of reference for the Autonomous workers in Ibero-America to identify the skills that are best paying through digital platforms of independent employment. Likewise, the academy and government are expected to consider these findings to align the training programs to the labor demand in the gig economy.

\section{Resumo}

Nos últimos anos, tem havido um aumento no uso de plataformas digitais de trabalho freelance (Freelancer.com 2015; 2019) como alternativa ao emprego tradicional. No entanto, há um conhecimento limitado sobre as opções de trabalho que os profissionais de TI têm dentro dessas plataformas. O objetivo deste documento é apresentar uma caracterização do mercado de trabalho dos profissionais das Tecnologias da Informação (TI) que trabalham na gig economy por meio da plataforma Workana. Para tanto, foram compiladas ofertas de emprego pertencentes à categoria "programação e tecnologia", publicadas entre agosto e dezembro de 2019 por empregadores localizados na Argentina, Brasil, Chile, Colômbia, México e Espanha. Foram levados em consideração o estado de desenvolvimento, escopo, orçamento, subcategoria e habilidades em cada oferta. Para conhecer as habilidades mais bem remuneradas, foram consideradas as ofertas associadas a 
uma única habilidade. Verificou-se que as habilidades mais requeridas pelos empregadores foram PHP, JavaScript, MySQL, Android, HTML, HTML5, Responsive Web Design, iPhone, CSS, API e WordPress. As habilidades mais bem remuneradas foram Android, C, React native e React.js. Espera-se que os resultados apresentados sirvam de referência para que os trabalhadores autônomos de TI na Ibero-América conheçam as habilidades mais bem pagas por meio das plataformas digitais de trabalho autônomo. Da mesma forma, espera-se que a academia e os governos considerem esses achados para alinhar os programas de treinamento à atual demanda de trabalho na gig economy.

\section{Introducción}

En 2018, por sexto año consecutivo, el trabajo por cuenta propia aumentó más que el empleo asalariado en Latinoamérica y el Caribe (CEPAL y OIT, 2019). Esto expone un detrimento de la calidad media del empleo remunerado en esa región y se comprende entonces el crecimiento del trabajo autónomo mediado por plataformas digitales. Tan sólo del año 2014 al año 2018 hubo un aumento de 17 millones de usuarios registrados en la plataforma freelancer.com (ibid., 2015; 2019) a nivel mundial; mientras que en la plataforma Workana se publicaron casi un millón de proyectos desde su fundación en el año 2012 y hasta el año 2019 (Workana, 2019a). En ambas plataformas la mayoría de los trabajadores contratados pertenecieron al área de TI y programación (Llorente, 2019; Workana, 2019b), esto advierte que los profesionales de TI están considerando la gig economy como una alternativa.

Los salarios que perciben los trabajadores se ven condicionados por variables como la industria, el tamaño de la empresa, las funciones del puesto, la antigüedad, la ubicación geográfica, entre otras (Global Knowledge, 2019). En el año 2019 el ingreso medio anual de los profesionales de TI en Estados Unidos fue de \$113.639 USD; mientras que en Latinoamérica fue de \$41.465 USD de acuerdo con Global Knowledge (2019). Contrastan significativamente los datos de la plataforma Empleos TI (2020), la cual indicó que durante el año 2019 en México los profesionales de TI tuvieron un ingreso promedio mensual de \$27.717 MXN —aproximadamente \$1.439,01 USD—. Así mismo Galván (2020) encontró que el salario mensual promedio de los profesionales de TI en México fue de \$41.499 MXN -aproximadamente $\$ 2.152,07$ USD-. Estos resultados se basaron en encuestas pero carecieron de una distinción entre el trabajo como freelance y el empleo tradicional.

Por su parte la plataforma Workana reportó que en el año 2019 el 46.9\% de los freelancers ganó menos de \$300 USD mensualmente y solo el 7.9\% tuvo ingresos mayores a \$1.500 USD por mes; a estos últimos les consideró como top freelancers.

Workana es una plataforma está enfocada al mercado de Latinoamérica, permite a los empleadores publicar ofertas de trabajo o de posiciones de largo plazo y posibilita a los freelancers postularse. Además, cuenta con un sistema de puntuación para poder valorar a 
los empleadores y freelancers de acuerdo con su desempeño. Respecto de los ingresos que percibe un freelancer a través de la plataforma Workana, se puede hacer solamente un retiro al mes por medio de "PayPal, Payoneer Tarjeta, Payoneer Transferencia, y Transferencia Bancaria Local (solo para Argentina y México)" (Workana, 2020b); estos métodos de retiro incluyen una comisión de hasta el $4 \%$ del valor a retirar.

En el año 2019 las ofertas de empleo en dicha plataforma estuvieron segmentadas de acuerdo a las categorías: diseño y multimedia; finanzas y negocios; ingeniería y tecnología, legal; marketing y ventas; redacción y traducción; soporte administrativo; programación y tecnología. Referente a esta última categoría, las tecnologías de la información se definen como el uso de hardware, software, servicios e infraestructura de soporte para administrar y entregar información usando voz, datos y video (North Dakota Information Technology Department, 2017); mientras que la programación está definida como la actividad de escribir programas de computadora (Cambridge University Press, 2021). La diferencia entre estos dos conceptos radica en que la programación es una aplicación específica de las tecnologías de la información.

La mencionada categoría programación y tecnología en Workana (2019c) se conformó por las siguientes subcategorías: aplicaciones de escritorio; diseño web; programación de apps. Android, iOS y otros; programación web; tiendas virtuales (ecommerce); WordPress; otros.

Dentro de Workana las ofertas de empleo se pudieron filtrar por categoría —el área del conocimiento a la que pertenece la oferta—, subcategoría —se muestran las categorías disponibles en el anexo de este trabajo-, habilidades — necesarias para cumplir con el trabajo-, fecha de publicación, modalidad del trabajo - precio fijo o precio por hora-, ubicación del empleador, historial del empleador y propuestas que ya se hayan hecho. Además, cada oferta de empleo expuso su presupuesto - intervalo del monto que pagará el empleador - tipo de empleo - ya sea proyecto o posición de largo plazo—, estado de desarrollo, alcance y puntuación del empleador (Workana, 2019c). Nuevas características fueron añadidas en el año 2020, que también se exponen en el anexo de este artículo.

Hay que destacar que la plataforma Workana (2020a) ofrece un plan de beneficios conformado por:

- Plan plus con un precio de \$5,18 USD al mes con los beneficios de añadir más habilidades en el perfil del freelancer, retirar ingresos dos veces mensualmente, hacer un examen de certificación de habilidades sin costo por mes y cinco contactos extra semanalmente. Un contacto en Workana (2020c) significa un mensaje que el freelancer puede enviar a un potencial cliente, lo que implicaría conocer mejor los requerimientos de este y poder adecuarse a sus necesidades para conseguir ser contratado.

- Plan professional con un costo mensual de \$16,60 USD que incuye: retiros ilimitados de ingresos, 15 contactos extra semanalmente y exámenes de certificación de habilidades ilimitados. 
-Plan premium que, a diferencia del plan Plan professional, ofrece más de 50 contactos extra a la semana y soporte premium por $\$ 23,35$ USD al mes.

Al indagar sobre qué valoran más los empleadores a través de la plataforma, se encontró que la experiencia del freelancer fue el atributo más importante al momento de contratar, de acuerdo con Workana (2019b). Sin embargo, se desconocen cuáles son las habilidades que más demandan los empleadores y las que mejor retribuyen a los freelancers a través de las plataformas digitales de trabajo gig como Workana.

Derivado de lo recién planteado, el objetivo de este artículo es presentar las habilidades mejor remuneradas a los profesionales de TI freelancers por los empleadores de Argentina, Brasil, Chile, Colombia, México y España dentro de la plataforma Workana. Para este fin se recopilaron ofertas de trabajo publicadas por empleadores de los países mencionados dentro de la plataforma Workana de agosto a diciembre de 2019. Posteriormente las ofertas de empleo fueron almacenadas en una base de datos relacional y a través de estadísticas descriptivas se encontraron las habilidades más demandadas, así como aquellas con las mayores retribuciones.

\section{Revisión de la bibliografía}

\section{Plataformas digitales de trabajo gig}

La Tecnologías de la Información y Comunicaciones (TIC) se han estado posicionando como el agente general de la producción y como uno de los procesos de formación en la economía digital (Berdykulova, 2014). Constantinescu (2017) señaló que la digitalización estaba provocando que los modelos de negocios, la perspectiva de las políticas y las normas sociales, se posicionaran como una herramienta estratégica. No obstante, Harteis (2018) indicó que la digitalización tendría como consecuencia que los procesos de trabajo cambien e incluso algunos entren en desuso y, dependiendo de esos cambios, se requerirá que en los lugares de trabajo se brinde capacitación.

El término gig economy se usa para referirse a aquellos trabajos que son esporádicos dados en una relación triangular entre un trabajador y un usuario final, como un individuo o un negocio (Zwick, 2018). Esa relación tiene como intermediario el servicio de una plataforma digital (Stewart y Stanford, 2017); tal es el caso de plataformas como Uber, Glovo, Didi, Upwork, Workana, Airbnb y BlaBlaCar. Entre los beneficios de las plataformas digitales se encuentra el poder encontrar a una persona que cumpla con las habilidades necesarias para cumplir con la tarea que requiere el negocio o individuo empleador. En este sentido, cabe mencionar que el anglicismo freelancer es utilizado para referirse a los trabajadores autónomos que por su propia cuenta buscan empleo e ingresos; tal como lo citó Jang (2017). El trabajo flexible en línea se caracteriza porque el ciclo de tiempo es corto y el trabajador tiene un alto nivel de control sobre los tiempos que empleará en realizar el trabajo (Lehdonvirta, 2018). De acuerdo con Graham (2019) los beneficios económicos serían 
significativos especialmente para los trabajadores que se encuentran en sitios donde es difícil conseguir buenos empleos. Donovan, Bradley y Shimabukuro (2016) opinaron que la gig economy puede ser contemplada como una expansión del trabajo independiente tradicional que puede facilitar el empleo puente o brindar oportunidades para generar ingresos cuando las circunstancias no se adaptan al empleo tradicional a tiempo completo y durante todo el año.

Ahora bien, es importante mencionar que el empleo como freelancer puede tener varias dificultades, como la cantidad de trabajos esporádicos que se deben atraer con tarifas competitivas, capacidad de organización y relaciones con clientes extranjeros (Roy y Shrivastava, 2020). Así mismo uno de los más grandes desafíos del trabajo como freelancer es la regulación sobre los derechos laborales para esta modalidad de trabajo. Por ejemplo Ruiz Tobón y Valencia Jiménez (2020) apuntaron que en Colombia uno de los mayores riesgos al trabajar como freelance es la limitada cobertura de seguridad social para este tipo de trabajadores. De Stefano (2015) sugirió que la gig economy debe incluirse en la economía y que es necesario defender los empleos gig para que logren ser reconocidos plenamente como trabajo y se pueda regular su actividad laboral. Es importante resaltar que, aunque ha habido avances en las regulaciones del trabajo autónomo, el desconocimiento de las leyes laborales facilita el incumplimiento de los derechos que todo trabajador tiene.

\section{Trabajos relacionados}

Graham, Hjorth y Lehdonvirta (2017) expusieron datos de las transacciones efectuadas durante marzo de 2013 en una de las plataformas más grandes de trabajo digital en un formato anonimizado para fines de protección de privacidad. Sus resultados indicaron que en Argentina, Brasil, Chile, Colombia, México y España la mayoría de los empleadores fueron extranjeros y la media de la tarifa que solicitaron los trabajadores osciló entre \$10 USD y \$20 USD por hora. Por su parte, Álvarez-Flores, Núñez-Gómez y OlivaresSantamarina (2018) recopilaron ofertas de trabajo en sitios de búsqueda de empleo con el fin de brindar una aproximación de la demanda laboral en la industria de la publicidad. En dichas ofertas de trabajo se identificaron palabras clave de la industria publicitaria para clasificar las ofertas de acuerdo al área laboral, sector publicitario e índole de la empresa. Los hallazgos muestran las competencias demandadas en el mercado laboral de la industria publicitaria, resaltando la necesidad de que los egresados tengan una formación transversal, entiendan el ecosistema digital y tengan dominio del idioma inglés.

Respecto a la disciplina de las TI, la plataforma de concursos de programación HackerRank (2020) expuso los resultados de su encuesta aplicada a 116 mil desarrolladores de 162 países. Sus hallazgos indicaron que la media salarial anual fue de \$54.491,33 USD. Perl fue el lenguaje que se asocia a la media más alta de salario, seguido de Scala y Go. De la misma forma, la comunidad de programadores Stack Overflow (2020) realizó una encuesta a cerca de 65 mil programadores en febrero de 2020. Sus resultados indicaron que a nivel mundial 
los profesionales que programaron con los lenguajes Go, Perl y Scala tuvieron los salarios más altos, con ingresos promedio de \$75.000 USD. Mientras que los roles mejor pagados fueron DevOps e ingeniería de confiabilidad de sitios. No obstante, en ambas encuestas los resultados no distinguieron el país de los encuestados. En México, la encuesta de Galván (2020) presentó que React, Electron y Xamarin fueron los frameworks mejor pagados en el año 2019. Por su parte, la encuesta efectuada por la plataforma Empleos TI (2020) indicó que los lenguajes de programación con los salarios más bajos en México fueron PHP, Cobol y Visual Basic.

En relación a las habilidades más demandadas en la gig economy, la plataforma Upwork reportó que .NET Core, TypeScript, Landing pages, eBooks y Android se posicionaron como las cinco habilidades más demandadas de julio a septiembre del año 2019 (Upwork, 2019). Específicamente en Latinoamérica, se puede tener una aproximación del mercado laboral de los profesionales de TI freelancers con base en el Reporte Workana 2019 (Workana, 2019b). En él se expone que, lo que más valoraron las empresas cuando trabajan con freelancers, fue su profesionalismo y adecuación a proyectos específicos. Sin embargo el reporte carece de una especificación respecto a las habilidades más demandadas en la plataforma.

\section{Metodología}

Se desarrolló un estudio exploratorio que tomó como base la metodología de Álvarez-Flores, Núñez-Gómez y Olivares-Santamarina (2018). Al igual que este último se recopilaron ofertas de empleo en plataformas digitales, en este caso se utilizaron ofertas de empleo freelance publicadas de agosto a diciembre de 2019 por empleadores situados en Argentina, Brasil, Chile, Colombia, México y España en la categoría programación y tecnología de Workana.

Se desarrolló una herramienta propia de software - con PHP versión 6- que generó un archivo HTML con el código fuente por cada página de Workana que mostraba las ofertas de empleo. Semejante a Graham, Marco y Myers (2018) se analizaron los archivos con ayuda de la herramienta desarrollada y se migraron los datos a una base de datos relacional por cada país con la estructura mostrada en la figura 1.

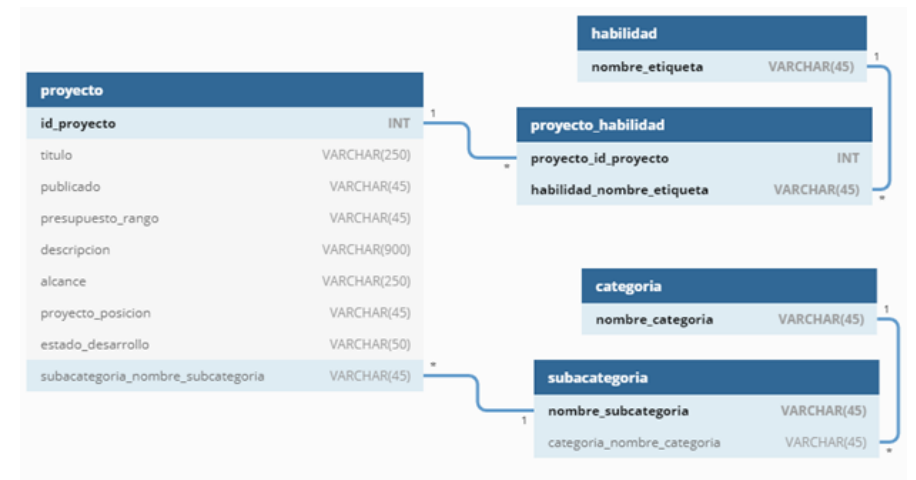

Figura 1: Base de datos: modelo relacional. 
Los nombres de las tablas creadas hacen referencia a los datos que almacenaron. En la tabla categoría se almacenó el nombre de la categoría de la publicación. La tabla subcategoría guardó el nombre de la subcategoría de la oferta de empleo y la categoría a la que pertenece. En la tabla proyecto se guardaron el número identificador de la oferta de empleo, el título, la fecha de publicación, el presupuesto que le asignó el empleador, la descripción del proyecto, el alcance del proyecto, el tipo de empleo al que corresponde la publicación y el estado de desarrollo de la tarea a realizar. La tabla habilidad almacenó, sin redundancia, todas las habilidades posibles que puede tener uno o varios proyectos. La tabla proyecto_habilidad generó una tupla que asocia la oferta de empleo con cada una de las habilidades - por separado— que el empleador consideró como habilidades necesarias para realizar el proyecto.

Posteriormente se efectuó una inspección para identificar las ofertas de trabajo con datos no pertinentes y se procedió a eliminarlas. Para evitar la redundancia de ofertas de trabajo, se identificaron aquellos registros que tuvieran el mismo título, presupuesto, descripción y habilidades. Una vez identificadas, se conservó sólo la oferta de empleo con la fecha de publicación más reciente.

\section{Análisis de los datos}

En este estudio fueron consideradas solamente las ofertas de trabajo que el empleador estableció como proyecto con presupuesto asignado también por proyecto. Las actividades a continuación descritas, se realizaron mediante consultas a la base de datos antes mencionada. A fin de reconocer el área de TI más demandada, se contaron las ofertas de trabajo existentes por cada subcategoría. Para identificar las habilidades más solicitadas por los empleadores se contabilizaron las habilidades, estado de desarrollo y alcance de las ofertas de trabajo.

Cabe mencionar que las ofertas estudiadas fueron recopiladas de Workana con presupuestos en dólares estadounidenses (USD) y en pesos mexicanos (MXN) incluso con empleadores situados fuera de América. Las ofertas de trabajo con presupuesto en pesos mexicanos se convirtieron a dólares estadounidenses. El tipo de cambio a usado fue el promedio del año 2019, que correspondió a \$19,26 MXN por \$1 USD (Banco de México, 2019). Para conocer las ofertas de trabajo mejor pagadas, se tomó como referencia el ingreso mensual de un top freelancer en Workana — \$1.500 USD—. Se seleccionaron entonces aquellas ofertas de trabajo que tuvieron alguno de los presupuestos mostrados en la tabla 1, porque en promedio fueron igual o mayor a \$1.500 USD.

Tabla 1. Presupuestos equivalentes a la ganancia mensual de un top freelancer en Workana. 


\begin{tabular}{|l|}
\hline Presupuesto por proyecto \\
\hline \hline USD $1.000-3.000$ \\
\hline \hline Más de USD 3.000 \\
\hline \hline MX\$ $20.000-60.000$ \\
\hline \hline MX\$ $19.000-60.000$ \\
\hline \hline Más de MX\$ 60.000 \\
\hline
\end{tabular}

Fuente: Elaboración propia con base en Workana (2019c).

A las ofertas de trabajo con presupuesto promedio mayor o igual a \$1.500 USD se les agrupó por subcategoría para registrar el área de TI a la que más pertenecieron tales ofertas. Posteriormente se hizo un conteo del número de proyectos que hubo por cada habilidad. De la misma forma se contaron las ofertas de trabajo existieron por cada estado de desarrollo y también se cuantificaron según su alcance.

Con el fin de conocer las habilidades de TI mejor remuneradas por los empleadores de Argentina, Brasil, Chile, Colombia, México y España, se seleccionaron las ofertas de trabajo que tenían una sola habilidad asociada. De esta manera se conoció el presupuesto que el empleador consideró pagar por una habilidad en específico. Posteriormente se calculó el promedio del presupuesto de las ofertas de trabajo. En caso de existir varios proyectos con la misma habilidad, se procedió a calcular el promedio del presupuesto de estos por cada país. Se eligieron solo las ofertas de trabajo con habilidades presentes en al menos dos países. Finalmente, se identificó el estado de desarrollo y alcance más presente en dichas ofertas de trabajo.

\section{Resultados}

El pago por proyecto estuvo presente en el $94.3 \%$ de las ofertas de empleo y el 5.7\% tuvieron el tipo de pago por hora. Por otro lado, fueron encontradas como proyecto el 98.18\% de las ofertas de trabajo con empleadores situados en Argentina, Brasil, Chile, Colombia, México y España. El resto de las ofertas correspondieron a una posición de largo plazo o se trataron de ofertas donde el empleador no asignó una clasificación. Se recolectaron 6374 ofertas que fueron publicadas entre agosto y diciembre de 2019, distribuidas como se muestra en la tabla 2 . 
Tabla 2: Ofertas de trabajo usadas para el análisis en Argentina, Brasil, Chile, Colombia, México y España.

\begin{tabular}{|l||l|}
\hline País & $\begin{array}{l}\text { Ofertas de trabajo } \\
\text { estudiadas }\end{array}$ \\
\hline \hline Argentina & 1011 \\
\hline \hline Brasil & 355 \\
\hline \hline Chile & 1250 \\
\hline \hline Colombia & 1129 \\
\hline \hline México & 1116 \\
\hline \hline España & 1058 \\
\hline
\end{tabular}

\section{Caracterización de las ofertas de trabajo}

Se consideró como área de TI a cada subcategoría perteneciente a la categoría programación y tecnología de Workana. La figura 2 muestra que en Argentina, Brasil, Chile, Colombia, México y España la mayoría de las ofertas de trabajo pertenecieron al área de TI programación web. Le siguió en menor proporción el área de TI programación de apps, Android, iOS y otros.
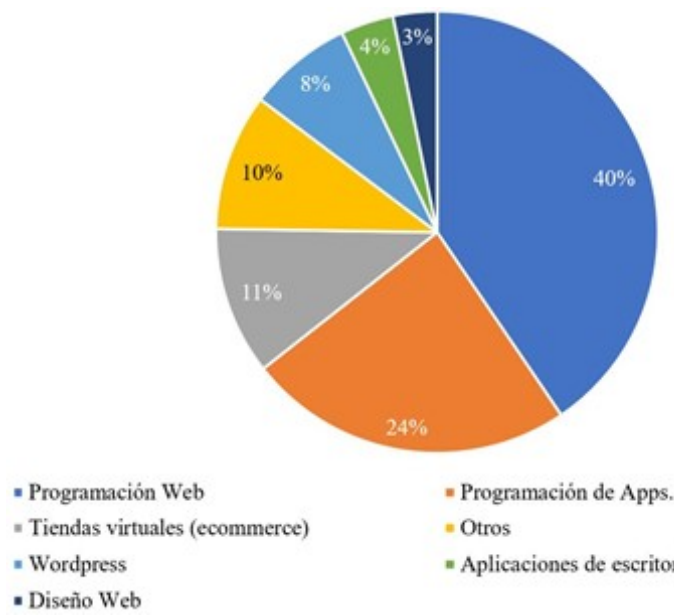

- Programación de Apps. Android, iOS y otros

- Otros

- Aplicaciones de escritorio

Figura 2: Distribución de las ofertas de trabajo publicadas por empleadores de Argentina, Brasil, Chile, Colombia, México y España, según área de TI. 
La figura 3 presentada a continuación expone las proporciones de las ofertas de trabajo según su estado de desarrollo. La mayoría de estas ofertas de trabajo ya tenía las especificaciones cuando se publicaron.

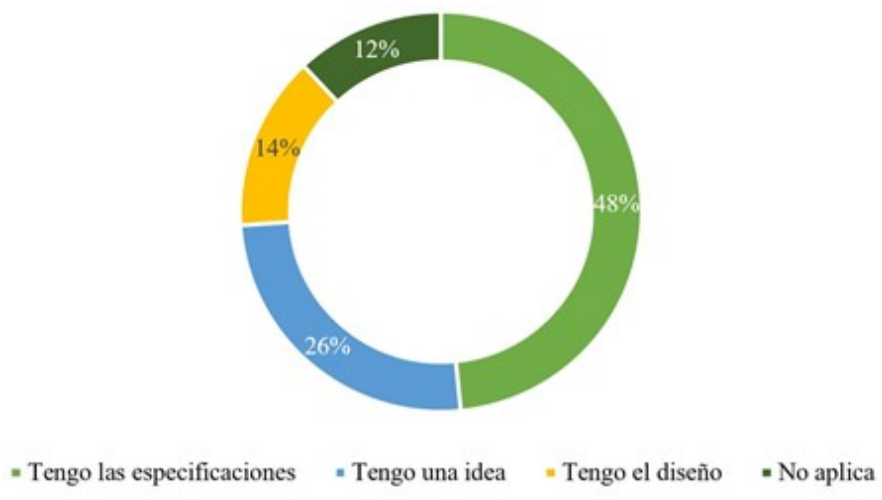

Figura 3: Distribución de las ofertas de trabajo publicadas por empleadores de Argentina, Brasil, Chile, Colombia, México y España, según estado de desarrollo.

De acuerdo con su alcance, las ofertas de trabajo fueron segmentadas y se exponen en la figura 4. En Argentina, Brasil, Chile, Colombia, México y España el alcance más presente fue realizar un cambio mediano, seguido de trabajar con un bug o cambio pequeño. Solo se descartó una oferta que no tuvo alcance asignado por el empleador.

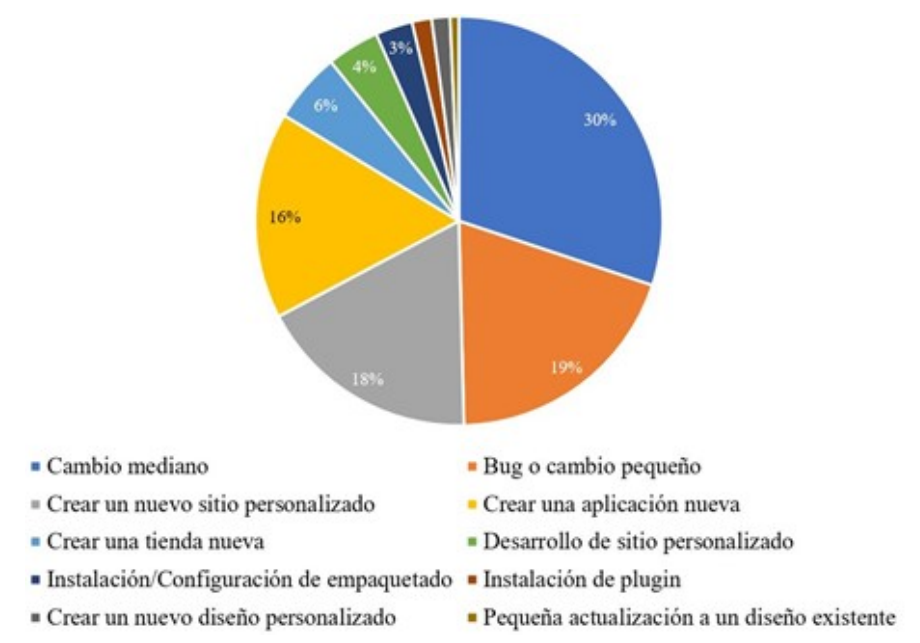

Figura 4: Distribución de las ofertas de trabajo publicadas por empleadores de Argentina, Brasil, Chile, Colombia, México y España, según alcance.

La tabla 3 muestra las habilidades que más se solicitaron en las ofertas de trabajo publicadas por empleadores situados en Argentina, Brasil, Chile, Colombia, México y España. Se incluyeron aquellas habilidades presentes en al menos mil proyectos. Las primeras posiciones fueron para PHP, JavaScript y MySQL.

Tabla 3: Habilidades más demandadas. 


\begin{tabular}{|l|l|}
\hline Habilidad & Proyectos \\
\hline \hline PHP & 3825 \\
\hline \hline JavaScript & 2861 \\
\hline \hline MySQL & 2795 \\
\hline \hline Android & 1893 \\
\hline \hline HTML5 & 1788 \\
\hline \hline HTML & 1691 \\
\hline \hline Responsive Web & 1380 \\
\hline \hline Design & 1187 \\
\hline \hline iPhone & 1278 \\
\hline \hline CSS & 1190 \\
\hline \hline WordPress & 1187 \\
\hline
\end{tabular}

Caracterización de las ofertas de proyectos con presupuesto promedio de $\$ 1.500$ USD o más

Fueron 1102 ofertas de trabajo con un presupuesto de al menos \$1.500 USD en Argentina, Brasil, Chile, Colombia, México y España. Se calculó el valor porcentual de estas ofertas con respecto al total de ofertas estudiadas en cada país. Los resultados se exponen en la tabla 4 , donde se observa que México tuvo la mayor proporción de ofertas de trabajo con presupuesto mínimo de $\$ 1.500$ USD. El segundo país con más ofertas de este tipo fue Colombia.

Tabla 4: Ofertas de trabajo con presupuesto igual o mayor a \$1.500 USD de empleadores 
de Argentina, Brasil, Chile, Colombia, México y España

\begin{tabular}{|l||l|l|}
\hline País & $\begin{array}{l}\text { Ofertas de trabajo con } \\
\text { presupuesto de \$1.500 USD o } \\
\text { más }\end{array}$ & $\begin{array}{l}\text { Valor porcentual del } \\
\text { total de ofertas de } \\
\text { trabajo }\end{array}$ \\
\hline \hline Argentina & 162 & $16.02 \%$ \\
\hline \hline Brasil & 47 & $13.24 \%$ \\
\hline \hline Chile & 229 & $18.32 \%$ \\
\hline \hline Colombia & 231 & $20.46 \%$ \\
\hline \hline España & 187 & $16.76 \%$ \\
\hline \hline México & 246 & $23.25 \%$ \\
\hline
\end{tabular}

En la tabla 5 se presenta la cuantía de las ofertas de trabajo por país y por área de TI. Para cada país se resaltaron las cifras en una escala de colores ascendente de blanco a azul. Se puede apreciar que, en la mayoría de los países, programación de apps, Android, iOS y otros fue el área de TI más solicitada. No así en España, que tuvo programación web como el área de TI más demandada.

Tabla 5: Distribución de las ofertas de trabajo con presupuesto igual o mayor a $\$ 1.500$ USD de empleadores de Argentina, Brasil, Chile, Colombia, México y España, según área de TI.

\begin{tabular}{lllllll}
\hline Área de TI & Argentina & Brasil & Chile & Colombia & España & México \\
\hline Aplicaciones de escritorio & 2 & 2 & 7 & 15 & 4 & 12 \\
Diseño web & 1 & & 3 & 7 & 1 & 2 \\
$\begin{array}{l}\text { Otros } \\
\text { Programación de apps }\end{array}$ & 10 & 2 & 12 & 9 & 21 & 10 \\
$\begin{array}{l}\text { Android, iOS y otros } \\
\text { Programación web }\end{array}$ & 50 & 24 & 119 & 107 & 59 & 123 \\
$\begin{array}{l}\text { Tiendas virtuales } \\
\text { (ecommerce) }\end{array}$ & 15 & 2 & 13 & 20 & 22 & 19 \\
\begin{tabular}{l} 
Wordpress \\
\hline
\end{tabular} & 2 & & 1 & 1 & 4 & 2 \\
\hline
\end{tabular}

La tabla 6 expone la cuantía de ofertas de trabajo según el estado de desarrollo que tenían cuando se publicaron desde Argentina, Brasil, Chile, Colombia, México y España. Se aplicó una escala de colores ascendente de blanco a azul por país. En la mayoría de los países el 
estado de desarrollo más concurrente fue tengo las especificaciones, a excepción de Brasil.

Tabla 6: Distribución de las ofertas de trabajo con presupuesto igual o mayor a \$1.500 USD de empleadores de Argentina, Brasil, Chile, Colombia, México y España, según estado de desarrollo.

\begin{tabular}{lllllll}
\hline Estado de desarrollo & Argentina & Brasil & Chile & Colombia & España & México \\
\hline No aplica & 11 & 7 & 14 & 10 & 19 & 26 \\
Tengo el diseño & 20 & 6 & 38 & 36 & 26 & 25 \\
Tengo las especificaciones & 88 & 16 & 110 & 121 & 97 & 116 \\
Tengo una idea & 43 & 18 & 67 & 64 & 45 & 79 \\
\hline
\end{tabular}

La distribución de las ofertas de trabajo por país según su alcance se expone en la tabla 7. En las columnas que presentan la proporción de las ofertas, se aplicó una escala ascendente de blanco a azul. Se puede apreciar que el alcance más presente fue crear una aplicación nueva, a excepción de España, donde el alcance más recurrente fue realizar un cambio mediano.

Tabla 7: Distribución de las ofertas de trabajo con presupuesto igual o mayor a \$1.500 USD de empleadores de Argentina, Brasil, Chile, Colombia, México y España, según alcance.

\begin{tabular}{|c|c|c|c|c|c|c|}
\hline Alcance & Argentina & Brasil & Chile & Colombia & España & México \\
\hline $\begin{array}{l}\text { Bug o cambio } \\
\text { pequeño }\end{array}$ & 9 & 11 & 5 & 3 & 8 & 7 \\
\hline Cambio mediano & 41 & 9 & 57 & 55 & 58 & 48 \\
\hline $\begin{array}{l}\text { Crear un nuevo diseño } \\
\text { personalizado }\end{array}$ & & & 2 & 3 & 3 & 2 \\
\hline $\begin{array}{l}\text { Crear un nuevo sitio } \\
\text { personalizado }\end{array}$ & 32 & 7 & 62 & 62 & 51 & 67 \\
\hline $\begin{array}{l}\text { Crear una aplicación } \\
\text { nueva }\end{array}$ & 64 & 17 & 93 & 92 & 47 & 102 \\
\hline $\begin{array}{l}\text { Crear una tienda } \\
\text { nueva }\end{array}$ & 12 & 2 & 8 & 14 & 14 & 14 \\
\hline $\begin{array}{l}\text { Desarrollo de sitio } \\
\text { personalizado }\end{array}$ & 1 & 1 & 1 & 1 & 5 & 3 \\
\hline $\begin{array}{l}\text { Instalación de plugin } \\
\text { Instalación }\end{array}$ & & & & & & 1 \\
\hline $\begin{array}{l}\text { Configuración de } \\
\text { empaquetado }\end{array}$ & 2 & & 1 & & 1 & 2 \\
\hline $\begin{array}{l}\text { Pequeña actualización } \\
\text { a un diseño existente }\end{array}$ & 1 & & & 1 & & \\
\hline
\end{tabular}

En figura 5 se presentan las habilidades con más afluencia en las ofertas de trabajo con presupuesto mayor o igual a \$1.500 USD y de empleadores situados en Argentina, Brasil, Chile, Colombia, México y España. En la generalidad de los países la habilidad más solicitada fue Android, empleada en el desarrollo para dispositivos móviles. Le siguieron JavaScript y PHP, que se usan para el desarrollo web. Cabe resaltar que PHP fue la habilidad más demandada en las ofertas de trabajo empleadores de España con presupuesto mínimo de \$1.500 USD. 


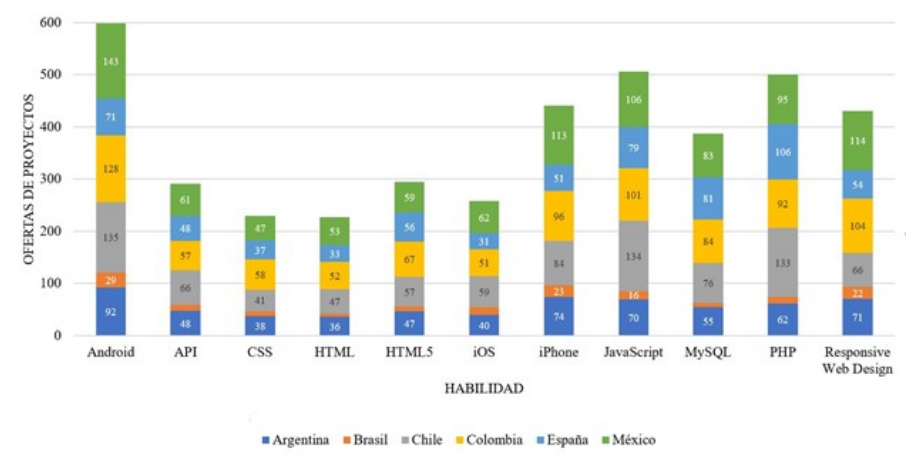

Figura 5: Habilidades más demandadas en las ofertas de trabajo con presupuesto igual o mayor a \$1.500 USD y con empleadores de Argentina, Brasil, Chile, Colombia, México y España.

\section{Caracterización de las ofertas de trabajo con una sola habilidad asociada y con presupuesto promedio de $\$ 1.500$ USD o más}

Las habilidades que se encontraron como mejor remuneradas fueron: Android, C, React Native y React.js. Cabe resaltar que Android fue la habilidad más demandada en proyectos con presupuesto de al menos de \$1.500 USD. Los resultados apuntan a que Android se pagaría mejor en Colombia, al igual que React.js. Por otro lado, $\mathrm{C}$ estaría mejor pagada en México. Mientras que React Native sería mejor retribuida en México y España. Lo anterior se puede observar en la figura 6 mostrada a continuación.

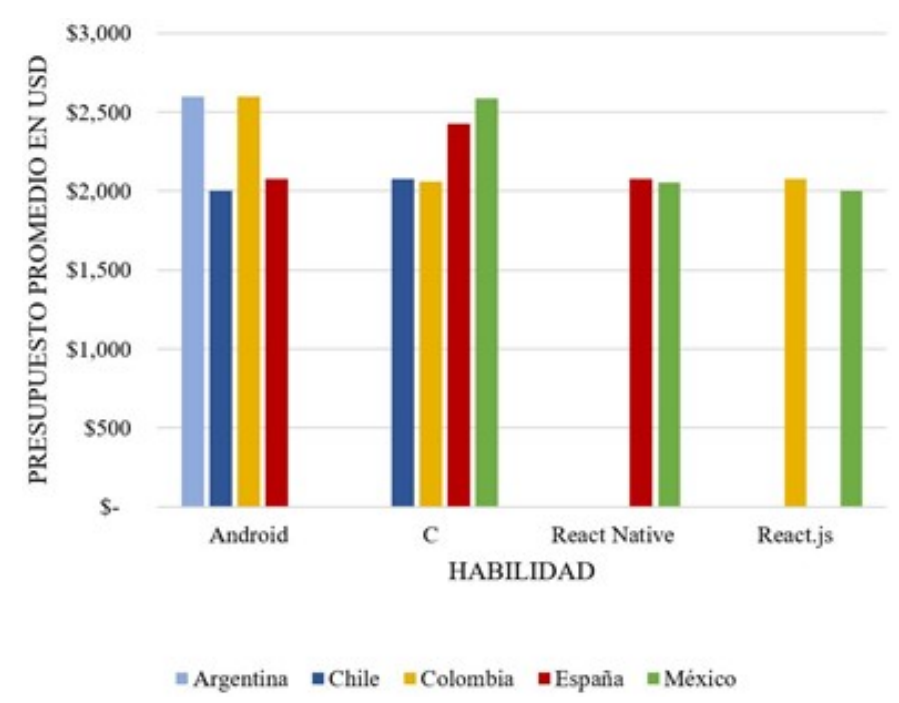

Figura 6: Habilidades mejor remuneradas en las ofertas de trabajo en Argentina, Brasil, Chile, Colombia, México y España.

Respecto al estado de desarrollo de las ofertas de trabajo con habilidades mejor remuneradas, el presupuesto promedio fue más alto cuando el estado de desarrollo fue tengo las especificaciones. Para la habilidad Android se observa que el presupuesto 
promedio fue aproximadamente $\$ 500$ USD mayor cuando se tuvieron las especificaciones, a comparación de cuando se tuvo una idea. Para la habilidad Facebook se notó un presupuesto promedio cerca de \$1.000 USD mayor cuando la oferta de empleo correspondió a una idea, en contraste de cuando se tuvo el diseño, como se aprecia en la figura 7.

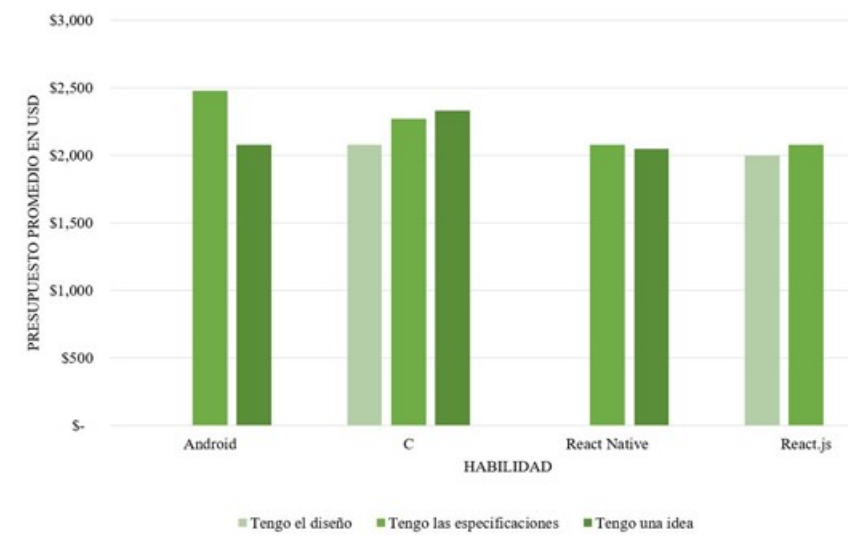

Figura 7: Estado de desarrollo de las ofertas de trabajo con habilidades mejor remuneradas en Argentina, Brasil, Chile, Colombia, México y España.

El presupuesto promedio de la habilidad C resultó más alto cuando el alcance fue crear un nuevo sitio personalizado, inmediatamente seguido del alcance cambio mediano. Para las habilidades mejor remuneradas, los diferentes alcances tuvieron variaciones menores a USD 60 por proyecto según se observa en la figura 8.

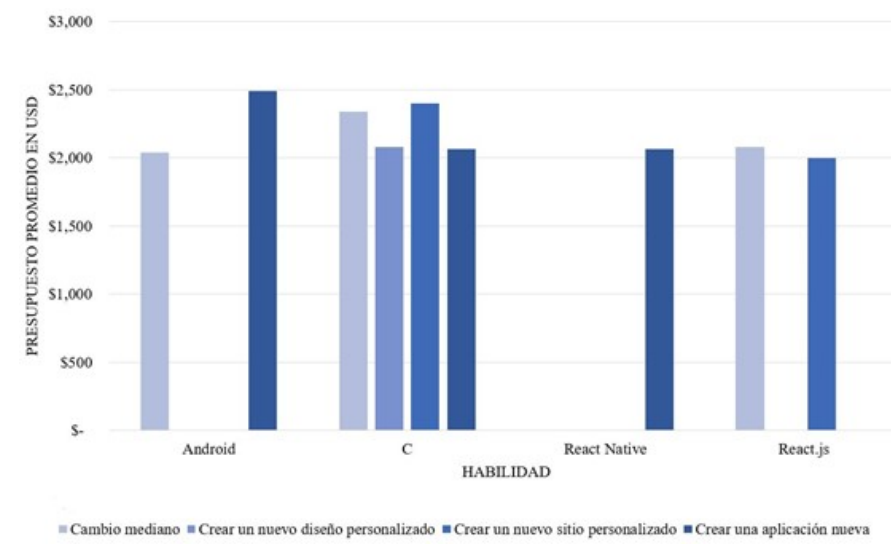

Figura 8: Alcance de las ofertas de trabajo con habilidades mejor remuneradas en Argentina, Brasil, Chile, Colombia, México y España.

\section{Conclusiones}

Este trabajo tuvo como propósito brindar una aproximación a las habilidades mejor remuneradas en Argentina, Brasil, Chile, Colombia, México y España. El estudio fue exploratorio y analizó ofertas de trabajo publicadas en la plataforma Workana. En dicha plataforma se pueden publicar ofertas para solicitar que un freelancer realice una tarea, la 
cual puede ser un proyecto o una cubrir una posición de largo plazo. Las ofertas de trabajo estudiadas fueron publicadas de agosto a diciembre de 2019 por empleadores situados en Argentina, Brasil, Chile, Colombia, México y España. Se contabilizó el número de ofertas de trabajo según su área de TI, estado de desarrollo y alcance.

Es importante resaltar que se ha identificado una barrera de entrada para emplearse a través de Workana, ya que los freelancers que pueden pagar por uno de los planes de beneficios (Workana, 2020a) se verán mejor posicionados ante los empleadores para ser seleccionados preferentemente.

Respecto a las ofertas de trabajo de los empleadores situados en Argentina, Brasil, Chile, Colombia, México y España se encontró que programación web fue el área de TI más demandada. Se descubrió que, cuando se solicitó un freelancer, el estado de desarrollo más presente en los proyectos fue "tengo las especificaciones". Así mismo se encontró que realizar un cambio mediano fue el alcance más solicitado en los proyectos. Las habilidades más demandadas fueron: PHP, JavaScript, MySQL, Android, HTML, HTML5, Responsive Web Design, iPhone, CSS, API y WordPress.

Conocer las habilidades más demandadas no implica necesariamente que sean las mejor pagadas. Por este motivo se consultaron las características de los proyectos con un presupuesto mayor o igual a \$1.500 USD —corresponde al ingreso mensual de un top freelancer (Workana, 2019a)—. Las habilidades más solicitadas en las ofertas de trabajo con presupuesto de al menos \$1.500 USD fueron: Android, JavaScript, PHP, iPhone, Responsive Web Design, MySQL, HTML, HTML5 y CSS. Android fue la habilidad más demandada en Argentina, Brasil, Chile, Colombia y México; en cambio en España PHP fue la habilidad más demandada dentro de las ofertas de trabajo con el presupuesto ya mencionado. Todas las habilidades corresponden a competencias relacionadas con el desarrollo web y móvil. Cuando los proyectos se ofertaron en Workana, la mayoría de estos ya tenían las especificaciones. Se descubrió que las ofertas de trabajo con el presupuesto de al menos \$1.500 USD buscaron tener como alcance crear una aplicación nueva; excepto en España, donde el alcance más recurrente fue crear un nuevo sitio personalizado.

En relación con las habilidades que se encontraron más demandadas, cabe decir que Android es el sistema operativo presente en más del 80\% de los teléfonos inteligentes del mundo (BBC News Mundo, 2019). Por otro lado, en el año 2018 se registraron ventas de 217 millones de iPhones en el mundo (MiniCreo, 2020). Los lenguajes PHP y JavaScript se caracterizan por ser usados para contener la parte lógica del software y generalmente se incrustan en HTML o HTML5. Estos dos últimos se refieren al lenguaje de marcado de hipertexto que sirve para visualizar contenido web. Ahora bien, el diseño web responsivo es un método para que el contenido web se adapte al tamaño del dispositivo donde se visualiza (Labrada Martínez y Salgado Ceballos, 2013). Por lo anterior, es natural que las habilidades encontradas se presenten en conjunto. Sin embargo, se desconoce cuánto se pagaría por cada una de las habilidades individualmente. Por ejemplo, el lenguaje PHP se posicionó 
como uno de los peores pagados en el Reporte del Mercado Laboral de TI 2020 (Empleos TI, 2020).

Con el fin de identificar el presupuesto promedio que pagarían los empleadores por una habilidad de manera individual, se seleccionaron proyectos con una sola habilidad asociada y con presupuesto mayor o igual a \$1.500 USD. Los hallazgos sugieren que, entre los freelancers, las habilidades mejor remuneradas por los empleadores situados en Argentina, Brasil, Chile, Colombia, México y España son: Android, C, React Native y React.js. Los presupuestos promedio más altos se presentaron cuando el estado de desarrollo fue tengo las especificaciones; así mismo cuando el alcance fue crear un nuevo sitio personalizado.

Este trabajo buscó contribuir a la bibliografía existente exponiendo las habilidades de TI mejor remuneradas y más demandadas con una metodología basada en ofertas de empleo que fueron obtenidas de la plataforma Workana. Se espera que el presente estudio sirva como recurso para replicar la metodología empleada y efectuar recurrentemente estudios del mercado laboral existente en las plataformas digitales de empleo independiente. En este sentido, los trabajadores autónomos pueden considerar emplearse usando las habilidades que se encontraron como las mejor remuneradas de acuerdo con los resultados recién expuestos. De igual forma, los hallazgos presentados pueden servir de apoyo para identificar las habilidades que más conviene aprender. Se espera que este trabajo ayude a las empresas para que conozcan la valoración de estas habilidades en la gig economy. Así mismo, se espera que este trabajo sirva como base para que los responsables del sector educativo alineen los programas de estudio del área de TI hacia las habilidades en tendencia dentro del mercado laboral digital.

\section{Discusión y trabajo futuro}

En función de lo hasta aquí planteado se puede argumentar que trabajar como freelancer en Argentina, Brasil, Chile, Colombia, México y España a través de una plataforma como Workana podría retribuir \$1.500 USD o más por un proyecto donde se empleen Android, C, React Native y React.js. Resalta que Android fue una de las cinco habilidades más demandadas en la plataforma de trabajo freelance Upwork durante el trimestre tercero del año 2019 (Upwork, 2019). Cabe mencionar que el framework React también fue encontrado como uno de los mejor retribuidos en México de acuerdo con Galván (2020). Destaca que las habilidades mejor pagadas dentro de la plataforma Workana fueron diferentes a aquellas asociadas con los salarios más elevados según las encuestas de HackerRank (2020) y Stack Overflow (2020).

Este estudio tuvo como alcance la recopilación de ofertas de proyectos publicadas en Workana por empleadores ubicados en Argentina, Brasil, Chile, Colombia, México y España. Como principal restricción se tuvo que la recolección de estas ofertas de proyectos se realizó dentro de una sola plataforma. Pese a que los rankings de mejores plataformas posicionaron a la plataforma freelancer.com como la más usada por los freelancers (Dinero 
en imagen, 2018; Llorens, 2016; Villarroel, 2018) se seleccionó la plataforma Workana debido a que en ella no es necesario ingresar a la publicación para conocer los requerimientos más importantes del proyecto. De igual manera, en Workana se pueden filtrar las ofertas de proyectos publicados por área del conocimiento y por país del empleador. Las ofertas de proyectos correspondieron exclusivamente al área del conocimiento programación y tecnología. Se han identificado como restricciones para el estudio que la descripción del proyecto es limitada y que no se puede tener más información sobre el empleador. Tampoco se puede comprobar si el freelancer seleccionado cumple realmente con las habilidades necesarias para llevar a cabo el proyecto. De igual manera, no es posible averiguar si el proyecto necesitaba habilidades diferentes a las que el empleador estableció. También se desconoce el criterio del empleador para establecer el presupuesto del proyecto y no se sabe si el empleador pagó un monto diferente al presupuesto que estimó cuando publicó el proyecto. Se tiene presente que, cuando se accedió a la plataforma Workana, fue probable que ciertas ofertas de proyectos hayan sido eliminadas previo a su recolección.

Se recomienda estudiar una mayor cantidad de plataformas digitales que median el trabajo freelance. Se sugiere ahondar en estudios que permitan conocer los criterios que influyen en el monto que paga el empleador a los freelancers y notar si hay contrastes con el pago que se realiza a los trabajadores con un empleo estándar. Resulta también necesario que en futuros estudios se pueda conocer el pago final que perciben los freelancers, especialmente por cada una de las habilidades más demandadas. Otro trabajo a futuro que se recomienda realizar es uno que contemple más información acerca del empleador. Así mismo, investigar la proporción de freelancers de Latinoamérica que son contratados por empleadores nacionales y extranjeros.

Se sugiere hacer un estudio cualitativo acerca de la oferta de freelancers para conocer qué es lo que valoran más y su experiencia en la migración al trabajo en línea. De igual forma, se propone analizar la evolución del mercado aplicando econometría. Finalmente se recomienda realizar un estudio de la evolución de las habilidades más demandadas y mejor remuneradas durante y posterior a la pandemia de enfermedad por COVID-19.

\section{Anexo}

\section{Nuevas características en ofertas de trabajo en Workana}

A partir del año 2020 las ofertas de trabajo en Workana (2019c) mostraron la duración aproximada del empleo, propuestas recibidas por el empleador, integraciones API, roles necesarios, método de pago del empleador — verificado o no verificado- y disponibilidad requerida. Así mismo, se incluyó la subcategoría data science en la categoría programación y tecnología. 


\section{Bibliografìa}

- Álvarez-Flores, E. P., Núñez-Gómez, P., \& Olivares-Santamarina, J. P. (2018). Perfiles profesionales y salidas laborales para graduados en Publicidad y Relaciones públicas: de la especialización a la hibridación. El profesional de la información (EPI), 27(1), 136-147.

- Banco de México, (2019). Tipo de cambio promedio del periodo. Sistema de Información Económica. Recuperado en 2019 de https://www.banxico.org.mx/SieInternet/consultarDirectorioInternetAction.do?sector=6\&ac cion $=$ consultarCuadro\&idCuadro $=\mathrm{CF} 86 \&$ locale $=\mathrm{es}$

- Berdykulova, G. M. K., Sailov, A. I. U., Kaliazhdarova, S. Y. K., \& Berdykulov, E. B. U. (2014). The emerging digital economy: case of Kazakhstan. Procedia-Social and Behavioral Sciences, 109, 1287-1291.

- BBC News Mundo. (5 de julio de 2019). Ni Google, ni Apple: ¿es posible encontrar un celular que no tenga nada que ver con estas dos compañías? BBC News Mundo. https://www.bbc.com/mundo/noticias-48870472

- Cambridge University Press. 10 de junio de 2021. Cambridge Dictionary. https://dictionary.cambridge.org/es-LA/dictionary/english/programming

- CEPAL, N. (2019). Coyuntura Laboral en América Latina y el Caribe. El futuro del trabajo en América Latina y el Caribe: antiguas y nuevas formas de empleo y los desafíos para la regulación laboral.

- Constantinescu, L. M. (2017). Digital Economy a Link to Competitiveness of Romania from Regional to Global. Revista de Management Comparat Internațional, 18(3), 326-342.

- De Stefano, V. (2015). The rise of the just-in-time workforce: On-demand work, crowdwork, and labor protection in the gig-economy. Comp. Lab. L. \& Pol'y J., 37, 471.

- Dinero en imagen. (11 de abril de 2020). 6 páginas con trabajos para freelancers en México

https://www.dineroenimagen.com/management/6-paginas-con-trabajos-para-freelances-en-m exico/92273

- Donovan, S. A., Bradley, D. H., \& Shimabukuru, J. O. (2016). What does the gig economy mean for workers?. http://digitalcommons.ilr.cornell.edu/key_workplace/1501

- Empleos TI. (2020). Reporte del Mercado Laboral de TI 2020 https://empleosti.com.mx/mercado-laboral-ti/2020

- Freelancer.com. (7 de enero de 2015). A Year in Freelancer.com: How Millennials are Changing the World.

http://press.freelancer.com.s3.amazonaws.com/Freelancer.com-2015-01-07-A-Year-In-Freela 
ncer.com-Millennials-Changing-World.pdf

- - - (15 de enero de 2019). Freelancer.com reveals the fastest growing online jobs in 2018. https://s3.amazonaws.com/press.freelancer.com/Freelancer.com reveals the fastest growing online jobs in 2018.pdf

- Galván, Pedro. (2020). Estudio de salarios SG 2020. Revista Software Guru. https://sg.com.mx/estudios/salarios/2020

- Global Knowledge. (2019). 2019 IT Skills and Salary Report.

https://www.globalknowledge.com/us-en/content/salary-report/it-skills-and-salary-report/\#re ports

- Graham, M. (Ed.). (2019). Digital economies at global margins. MIT Press.

- Graham, M., Hjorth, I., \& Lehdonvirta, V. (2017). Digital labour and development: impacts of global digital labour platforms and the gig economy on worker livelihoods. Transfer: European review of labour and research, 23(2), 135-162.

- Graham, S. J., Marco, A. C., \& Myers, A. F. (2018). Patent transactions in the marketplace: Lessons from the uspto patent assignment dataset. Journal of Economics \& Management Strategy, 27(3), 343-371.

- HackerRank. (2020). HackerRank 2020 Developer Skills Report. https://research.hackerrank.com/developer-skills/2020

- Harteis, C. (2018). Machines, change and work: An educational view on the digitalization of work. In The impact of digitalization in the workplace (pp. 1-10). Springer, Cham.

- Jang, J. (2017). The emergence of freelancer cooperatives in South Korea. Annals of public and cooperative economics, 88(1), 75-89.

- Labrada Martínez, E., \& Salgado Ceballos, C. (2013). Diseño web adaptativo o responsivo.

- Lehdonvirta, V. (2018). Flexibility in the gig economy: managing time on three online piecework platforms. New Technology, Work and Employment, 33(1), 13-29.

- Llorens, Georgy. (2016). 10 plataformas para emprender como freelancer. Entrepreneur. https://www.entrepreneur.com/article/276673

- Llorente, Analía. (27 de agosto de 2019). Freelancers: los empleos en internet para trabajadores autónomos más demandados en América Latina. BBC News Mundo. https://www.bbc.com/mundo/noticias-49463795

- MiniCreo. (2020). How Many iPhones Are There In History [To Date April 2020]. Mac Malware App List. https://www.minicreo.com/news/how-many-iphones-are-there.html - North Dakota Information Technology Department. (2017). Definition of Information 
Technology. Recuperado el 10 de junio de 2021 de https://www.nd.gov/itd/about-us/definition-information-technology

- Roy, G., \& Shrivastava, A. K. (2020). Future of Gig Economy: Opportunities and Challenges. IMI Konnect, 9, 14-25.

- Ruiz Tobón, J., y Valencia Jiménez, M. (2020) Nuevos retos de la legislación colombiana en el sistema de Seguridad Social Integral para los Trabajadores Digitales. [Monografía para optar por el título de abogadas]. Eafit.

- Schwab, K. (2019). The Global Competitiveness Report 2019. WEF.

http://www3.weforum.org/docs/WEF_TheGlobalCompetitivenessReport2019.pdf

- Stack Overflow. (2020). 2020 Developer Survey. https://insights.stackoverflow.com/survey/2020

- Stewart, A., \& Stanford, J. (2017). Regulating work in the gig economy: What are the options?. The Economic and Labour Relations Review, 28(3), 420-437.

- Upwork. (19 de noviembre de 2019). Upwork debuts The Upwork 100, ranking the top 100 in-demand skills for independent professionals.

https://www.upwork.com/press/releases/the-upwork-100-q3-2019

- Villarroel, Luis. (3 de octubre de 2018). 10 mejores plataformas para encontrar trabajo freelancer desde casa. Diario Freelancer.

https://diariofreelancer.com/10-mejores-plataformas-para-encontrar-trabajo-freelancer-desd e-casa/

- Workana. (2019a). ¿Qué es Workana?

https://www.workana.com/i/glosario/que-es-workana/

-_-. (2019b). Reporte Workana 2019.

https://reporte.workana.com/reporte/reporte-analisis-completo.pdf

-_- (2019c). Trabajos Freelance: Programación y Tecnología.

https://www.workana.com/jobs?ref=menu_projects_index

-_—. (2020a). ¿Qué son los planes de beneficios?

https://help.workana.com/hc/es/articles/360041612574-Qué-son-los-Planes-de-Beneficios-

-_- (2020b). ¿Qué métodos de retiro de dinero puedo elegir?

https://help.workana.com/hc/es/articles/360041937573-Qué-métodos-de-retiro-de-dinero-pu edo-elegir-

-_- (2020c). ¿Cómo funciona Workana?

https://www.workana.com/es/how-it-works/freelancer

- Zwick, A. (2018). Welcome to the Gig Economy: Neoliberal industrial relations and the 
case of Uber. GeoJournal, 83(4), 679-691.

\section{Biografía de las Autoras}

Edna-Patricia Santiago-Vargas: Ingeniera de software y Maestra en planeación estratégica y dirección de tecnología egresada de UPAEP Universidad, con estudios en la Universidad Politécnica de Madrid. Actualmente es Subdirectora de Sistemas Administrativos en la Secretaría de Comunicaciones y Transportes. Sus intereses de investigación son transformación digital del mercado laboral y economía de plataformas.

Catalina Ovando: Ingeniera en Electrónica y Comunicaciones por la Universidad Iberoamericana y Doctora en Telecomunicaciones por la Universidad Politécnica de Madrid. Actualmente es Titular de la Unidad de Tecnologías de Información y Comunicaciones en la Secretaría de Comunicaciones y Transportes. Es miembro del Sistema Nacional de Investigadores (SNI) nivel I y desde 2015 es docente en los posgrados de Planeación Estratégica y Dirección de Tecnología en UPAEP Universidad. En 2020 fue nombrada como miembro del Quinto Consejo Consultivo del Instituto Federal de Telecomunicaciones. Desde Marzo de 2021 es Vicepresidenta del capítulo IEEE ComSoc Puebla. Sus líneas de investigación son inclusión digital, adopción de la tecnología, políticas públicas de innovación y telecomunicaciones, plataformas digitales y su impacto en los sectores productivos. 